\title{
DNA Damage, Signaling and Repair: Protecting genomic integrity and reducing the risk of human disease
}

DNA is the genetic material that contains the "instructions" to not only guide the continuation of life but also govern the development, metabolism and functioning of living organisms. DNA damage occurs daily due to various exogenous (environmental) genotoxic agents and normal metabolic processes within cells. Even under normal growth and metabolic conditions, DNA damage occurs at a rate of approximately 10000 lesions per cell per hour in mammalian cells. DNA damage that is not properly and promptly repaired can lead to genomic instability, an increased likelihood of gene mutation or even cell death. Cells cannot function normally if DNA damage corrupts the integrity and accessibility of the essential information in the genome, which in turn has a harmful effect on health, such as carcinogenesis, tissue-degeneration or functional failure and accelerated aging. In this special topic, Chinese Science Bulletin presents a series of research articles and reviews covering some of the critical aspects of the molecular mechanisms of DNA damage signaling, repair and cellular responses.

DNA repair and the cell cycle checkpoint are two fundamental mechanisms governing genome integrity, and both are tightly interconnected in their signaling pathways, sharing some signaling molecules. Depending on the lesion inflicted on the DNA double helical structure, multiple repair pathways have evolved to replace the lost information and recover genomic integrity. The mechanistic pathways of repairing the damage occurring at one of the two helical domains of DNA include: (1) Direct reversal of the damage, which is the specialized mechanism for reversing a specific type of damage; for example, methyl guanine methyl transferase (MGMT) specifically removes a methyl group from DNA bases. (2) Excision repair, in which the damaged nucleotide is removed and the normal DNA structure is recovered using information from the undamaged strand. Excision repair mechanisms include base excision repair that repairs simple damage due to alkylation or deamination, and nucleotide excision repair that repairs bulky, complex or helix-distorting damage. In eukaryotic cells, there are two subpathways of nucleotide excision repair: global-genome nucleotide excision repair and transcription-coupled repair. Transcription-coupled repair can remove bulky DNA lesions located on the transcribed strand of an active gene more rapidly than those on the non-transcribed strand or in the general genomic DNA. And (3) mismatch repair, which corrects errors of DNA replication, recombination and induced mismatched bases. Obviously, DNA double-strand breakage (DSB) is a particularly hazardous type of DNA damage to dividing cells as it involves a break to both strands nearby in the double helix. There are two interconnected and collaborative pathways to repair this damage: homologous recombination and non-homologous endjoining.

Evoking the cell cycle checkpoints is one of the cellular mechanisms to maintain genomic integrity; this arrests the cell cycle until the DNA damage has been properly repaired, or all the chromosomes have been completely replicated and correctly aligned on the spindle. The checkpoints are typically classified into two categories: DNA damage checkpoints, including the G1/S, S-phase and G2/M checkpoints, and the spindle-structure checkpoint. The cell cycle checkpoint kinase Chk1 is a key mediator linking DNA damage to the cell cycle control mechanisms. When DNA damage is induced by ultraviolet light or replication stress, Chk1 is phosphorylated by the ataxia telangiectasia mutated (ATM) or ATM Rad3 related (ATR) kinases. Activated Chk1 phosphorylates and inactivates Cdc25C, preventing the transition from G2 to M phase. Translocation from the cytoplasm into the nucleus is necessary for Chk1 to function as a cell cycle checkpoint regulator. Chk1 contains two structural domains: a kinase domain in the N-terminal half and a regulatory domain in the C-terminal half of the protein. In this topic, Ning et al. [1] report that DNA damage-induced nuclear accumulation of Chk1 is tightly regulated by the motif between AA334-AA368 within its C-terminal regulatory domain. These authors described that the enzymatic activity and DNA repair capability of a truncated Chk1 mutant lacking the 108 C-terminal amino acids (368-476) was higher than that of full-length Chk1, while a truncated mutant lacking the 188 C-terminal amino acids (288-476) was almost devoid of enzymatic activity. However, the truncated mutant missing the 142 C-terminal amino acids (334-476), was equivalent to that of full-length Chk1. Ning et al. [1] have revealed that the regulatory domain of Chk1 contains both inhibitory and regulatory elements. The cyclin-dependent kinase inhibitor $\mathrm{p} 21^{\mathrm{WAF} 1 / \mathrm{Cip} 1}$ (also known as cyclin-dependent kinase inhibitor 1A, CDKN1A) 
is another critical regulator of the cell cycle and DNA damage response. It participates in multiple fundamental cellular processes, including cell cycle progression, apoptosis, transcription induction and DNA repair. p2 $1^{\text {WAF1/Cip1 }}$ itself is regulated at the levels of transcription, stability and/or subcellular translocation when DNA is damaged. Here, Cao et al. [2] report that promyelocytic leukemia protein (PML) contributes to the upregulation of p21 in a p53-independent pathway. PML inhibited the proteasome-mediated proteolysis of p21. Knock-down of PML resulted in a decreased association between p21 and proliferating cell nuclear antigen (PCNA), and decreased the repair of $\gamma$-irradiation-induced DSBs. Overexpression of p21 rescued the decreased efficiency of DNA DSB repair mediated by PML depression.

Post-translation modification, including phosphorylation, acetylation, poly(ADP-ribose) polymerization, ubiquitination and sumoylation, is an important means to modulate the activity of most of the DNA damage response (DDR) proteins. p53 plays a vital role in DDR, and its expression level or degradation is tightly regulated by the murine double minute 2 (MDM2)-mediated ubiquitination. The Smad ubiquitylation regulatory factor 1/2 (Smurf1/2) promotes p53 degradation by interacting with and stabilizing MDM2. Nie et al. [3] further report that, in cells in which DNA damage has been induced by etoposide, Smurf1 dissociates from MDM2, resulting in MDM2 destabilization and p53 accumulation.

Phosphorylation is the most common post-translation modification that occurs in DDR proteins during DNA damage signaling. A balance between phosphorylation and dephosphorylation is very important to maintain the proper activity of these DDR proteins. Liu and $\mathrm{Xu}$ [4] provide cutting-edge information on serine/threonine protein phosphatases involved in the DNA damage response. They propose that in situ dephosphorylation of phosphoproteins by protein phosphatases, instead of proteasome-mediated degradation, is the primary cellular mechanism.

Chromatin relaxation is a fundamental molecular event in response to DNA damage, and is necessary for access of the DDR proteins to the sites of damage. KRAB-associated protein (KAP-1), also known as tripartite motif-containing 28 (TRIM28), is a critical effector in ATM-dependent chromatin relaxation in response to DNA DSBs induced by ionizing radiation (IR). Qin et al. [5] report that p53-inducible gene 3 (PIG3), also known as tumor protein p53-inducible protein 3 (TP53I3), formed foci in the nucleus after IR-induced DNA DSB, and that this protein interacted and partially colocalized with phospho-KAP-1 foci. PIG3 must play a role in large-scale chromatin relaxation in DNA damage signaling. Moreover, the expression level of PIG3 was remarkably upregulated in a dose-dependent manner after IR exposure, and this radiation-induced expression of PIG3 was dependent on p53 status.

Epidermal growth factor receptor (EGFR) is frequently dysregulated in various human cancers. Overexpression and amplification of wild-type EGFR, or expression of mutated EGFR, is closely associated with cancer progression and a poor clinical outcome. In addition, augmented activation of EGFR results in an increased resistance to cancer radiotherapy and particular DNA-damaging chemotherapies. It is well known that EGFR controls diverse cellular events including proliferation, differentiation, cell cycle progression, survival (apoptosis) and transformation by stimulating a cascade of signal transduction pathways including the RAS/MAPK, JAK/STAT, PKC and PI3-K/AKT pathways. Importantly, EGFR signaling has now been revealed to function in DNA damage signaling and repair (see the review by Lu et al. [6]). In response to DNA damage signaling, EGFR is activated and translocated into the nucleus. Increasing evidence suggests that there are multiple ways through which EGFR and/or its downstream signaling effectors may alter the elements of base excision repair, non-homologous end-joining and homologous recombination, thereby governing DNA repair. It is likely that the mechanism of EGFR-regulated DNA repair is complex and that there are multiple interactions between the EGFR signaling effectors and the components of the DNA repair pathways. The review by Lu et al. [6] highlight the current knowledge regarding the role and mechanisms of EGFR in DNA repair, and provide important insights into the intrinsic regulatory networks of DNA damage repair.

The linking of RNA damage to human disease processes has gradually attracted much attention. Although we still know little about genotoxin-induced RNA damage compared with our knowledge of DNA damage, RNA damage can clearly lead to cell death and can be involved in the pathogenesis of degenerative diseases such as Alzheimer's disease and Parkinson's syndrome. To investigate the features of RNA damage precisely, it is necessary to establish a reliable and sensitive RNA damage-detection method. In this special topic, Tie et al. [7] report a novel technology to detect RNA strand breaks based on 3 '-end poly(A)-tailing PCR. Using this novel method, Tie et al. [7] successfully detected IR-induced RNA breaks in a number of DNA damage response genes, including ABL1, TP53, GADD45A and ATR.

Finally, this special topic, DNA Damage, Signaling and Repair, covers some of the most important topics linked to the mechanisms of DDR, providing an up-to-date account of the rapid progress in this expanding research field. I hope you enjoy it.

1 Ning L F, Wang H P, San D, et al. C-terminal domain of Chk1 regulates its subcellular location and kinase activity for DNA repair. Chinese Sci Bull, 2011, 56: 3138-3147

2 Cao L, Song Y, Tian B L, et al. PML contributes to p53-independent p21 up-regulation in gamma-irradiation induced DNA damage responses. Chinese Sci Bull, 2011, 56: 3148-3154

3 Nie J, Liu L, Zhao X H, et al. DNA damage stress induces the dissociation of Smurf1/2 from MDM2 in a slow manner. Chinese Sci Bull, 2011, 56: 
$3155-3161$

4 Liu B, Xu X Z. Serine/threonine protein phosphatases in DNA damage response. Chinese Sci Bull, 2011, 56: 3122-3131

5 Qin X, Zhang S M, Li B, et al. p53-dependent upregulation of PIG3 transcription by $\gamma$-ray irradiation and its interaction with KAP1 in responding to DNA damage. Chinese Sci Bull, 2011, 56: 3162-3171

6 Lu J C, Yang L F, Tao Y G, et al. Role of epidermal growth factor receptor in DNA damage repair. Chinese Sci Bull, 2011, 56: 3132-3137

7 Tie Y, Hu Z, Lv G X, et al. A novel method for ionizing radiation-induced RNA damage detection by poly(A)-tailing RT-PCR. Chinese Sci Bull, 2011, 56: $3172-3177$

Special Topic Editor

ZHOU PingKun

Department of Radiation Toxicology and Oncology, Beijing Institute of Radiation Medicine, Beijing 100850, China

Open Access This article is distributed under the terms of the Creative Commons Attribution License which permits any use, distribution, and reproduction in any medium, provided the original author(s) and source are credited. 